\title{
Sudden death in children with cardiac allograft vasculopathy
}

\author{
Borah J. Hong $^{1}$, S. Kristen Sexson Tejtel ${ }^{2}$, Aamir Jeewa ${ }^{3}$, Antonio G. Cabrera ${ }^{2}$ Jack F. Price $^{2}$, Jeffrey S. Heinle ${ }^{4}$, William J. Dreyer ${ }^{2}$ and Susan \\ W. Denfield ${ }^{2}$
}

${ }^{1}$ Division of Pediatric Cardiology, Department of Pediatrics, Seattle Children's Hospital, Seattle, Washington, USA

${ }^{2}$ Lillie Frank Abercrombie Section of Cardiology, Department of Pediatrics, Baylor College of Medicine, Houston, Texas, USA

${ }^{3}$ Division of Pediatric Cardiology, Department of Pediatrics, The Hospital for Sick Children, Toronto, Ontario, Canada

${ }^{4}$ Division of Congenital Heart Surgery, Department of Surgery, Baylor College of Medicine, Houston, Texas, USA

\begin{abstract}
Cardiac allograft vasculopathy (CAV) is a leading cause of late mortality in pediatric heart transplant patients. Sudden death (SD) is a known phenomenon in CAV. This study aims to characterize factors associated with SD versus heart failure death (HFD) in pediatric patients with CAV. This single center retrospective review identified 37 pediatric patients who died or were retransplanted as a result of CAV. $9(24 \%)$ of those patients died suddenly. There were no differences in rejection episodes between SD patients and HFD/retransplant patients. Ejection fraction (EF) was significantly higher in the SD group, median EF $62 \%$ versus $50 \%$ in the $\mathrm{HFD} /$ retransplant group $(p=0.045)$. Hemodynamics also differed, with a median right ventricular end diastolic pressure of 8 mmHg in the SD group versus 14 $\mathrm{mmHg}$ in the $\mathrm{HFD} /$ retransplant group $(p=0.011)$ and a median pulmonary capillary wedge pressure of $9 \mathrm{mmHg}$ in the $\mathrm{SD}$ group versus $14 \mathrm{mmHg}$ in the $\mathrm{HFD} /$ retransplant group $(p=0.027)$. Brain natriuretic peptide (BNP) was lower in the SD group, median $260 \mathrm{pg} / \mathrm{ml}$ versus $1081 \mathrm{pg} / \mathrm{ml}$ in the HFD/retransplant group $(p=0.013)$. SD patients were more likely to have lower BNP levels, normal hemodynamics, and normal systolic function, none of which should be reassuring in the setting of CAV.
\end{abstract}

\section{Introduction}

Cardiac allograft vasculopathy (CAV) is a leading cause of late mortality in pediatric heart transplant recipients [1]. Survival is approximately $50-60 \%$ at 3 years following the diagnosis of CAV depending on the age at transplant [1]. The only definitive therapy for CAV is retransplantation, but given the limited donor pool, the decision of when to list a CAV patient for retransplantation becomes crucial. Listing a pediatric patient with CAV who has evidence of graft failure and demonstrates symptoms of heart failure is a Class I indication [2]. However, there is a significant subset of patients with CAV who die suddenly and unexpectedly. Adult studies estimate that up to $40 \%$ of heart transplant recipients who die suddenly had evidence of CAV [3]. Those patients with CAV who also had left ventricular dysfunction and severe CAV with involvement of all three major vessels were at greatest risk of sudden death [4]. There have been attempts at primary prevention in these patients with implantable cardioverter-defibrillators (ICD), but the terminal rhythm is usually asystole or pulseless electrical activity in the majority of patients who die suddenly, therefore the benefit is unclear $[5,6]$.

The data in the pediatric heart transplant population is more limited, although there have been registry studies demonstrating that sudden death occurred in $16 \%$ of all heart transplant deaths in children [7]. That study did not find an association between CAV and sudden death, but the study authors concluded that the limitations of the registry data collection itself, as well as the low prevalence of CAV in that study cohort may have contributed to the inability to find an association [7]. A more recent study from the Pediatric Heart Transplant Study data demonstrated that the presence of an ejection fraction (EF) of $<45 \%$, a right atrial pressure of $>12 \mathrm{mmHg}$, or a pulmonary capillary wedge pressure $(\mathrm{PCWP})>15 \mathrm{mmHg}$ put children at increased risk of graft loss despite mild angiographic CAV [8]. However, this study did not stratify graft loss into sudden death versus heart failure death. Little is known if there are clinical or hemodynamic risk factors that can identify those pediatric patients with CAV who are likely to die suddenly. This study aims to identify characteristics of patients with CAV who die suddenly versus those who experience heart failure death, which may affect timing of listing for retransplant and improve patient survival.

\section{Methods}

This was a single center retrospective review of all pediatric patients who were transplanted between 1984 and 2012. Institutional review board approval was obtained. Inclusion criteria included all patients who were diagnosed with CAV by one of three methods: coronary angiography, explant pathology, or autopsy pathology and either died or underwent retransplantation. Those patients who had incomplete clinical records were excluded. Sudden death (SD) was defined as an out of hospital cardiac arrest that was unexpected in timing and mode of death. Heart failure death (HFD) was defined as patients with clinical

Correspondence to: Susan W. Denfield, Texas Children's Hospital, 6621 Fannin Street, MC-19345-C, Houston, TX 77030-2399, Tel: 832-826-5659; Fax: 832825-5899; Email: denfield@bcm.edu

Key words: cardiac allograft vasculopathy, pediatric cardiac transplantation, retransplantation, sudden death

Received: October 17, 2016; Accepted: November 19, 2016; Published: November 24, 2016 
evidence of poor cardiac function, requiring inotropes or mechanical circulatory support at the time of death. We chose to include and analyze the HFD and retransplantation patients in the same group to compare to our primary outcome group, the SD patients. The patients who were retransplanted had heart failure as their indication for listing, thus they were included as a heart failure death.

Patient demographics, primary cardiac diagnosis, and clinical information including cause of death were reviewed. Risk factors examined included number of episodes of endomyocardial biopsy confirmed acute cellular rejection (ACR) with grading of $2 \mathrm{R}$ or higher on the 2005 revised ISHLT scale or 5 or greater (equivalent to $\geq 2 \mathrm{R}$ ) on the older Texas Heart Institute scale [9] and antibody mediated rejection (AMR) based on histopathology and C4d immunohistochemistry staining, diagnosis of congenital heart disease or primary cardiomyopathy, systolic function as measured by echocardiogram with Simpson's biplane method for ejection fraction, cardiac catheterization hemodynamics, presence of implantable cardiac defibrillators (ICD) or pacemakers, previous arrhythmias, and clinical serum biomarkers including serum creatinine and brain natriuretic peptide (BNP) levels.

\section{Statistical analysis}

Patient characteristics are described as number and percentage as well as median and range, where appropriate. The primary clinical end point is mode of death (sudden death, heart failure death, or organ death as defined by retransplantation). Comparison of categorical variables between the SD patients and HFD/retransplant patients was performed using the Chi-square test where appropriate. Comparison of continuous variables between the SD patients and HFD/retransplant was performed using the Mann-Whitney $U$ test where appropriate. Standard Kaplan-Meier survival analysis was performed comparing the SD group to the HFD/retransplant groups. A value of $p<0.05$ was considered statistically significant. All statistical analyses were performed using IBM SPSS Statistics Version 18.0.

\section{Results}

\section{Patient characteristics}

From 1984 through 2012, there were 274 pediatric patients who underwent heart transplantation at our single institution. Three patients were excluded for incomplete data or if they were lost to follow up. Of the remaining 271 patients, we identified 54 (20\%) patients who had a diagnosis of CAV, and 40 were identified as having died or undergone retransplantation. Three of those patients died of noncardiac causes while 37 of them died from a cardiac etiology. Nine of the 37 patients died suddenly, 12 patients died of end stage heart failure, and 16 patients were retransplanted for graft dysfunction (Figure 1). Of the SD patients, 3/9 (33\%) were diagnosed at autopsy with prior coronary angiography interpreted as normal within the prior 2,6 , and 11 months, respectively. Of those who died of heart failure, 3/12 (25\%) were diagnosed at autopsy with one patient never having had coronary angiography due to small size, one patient having normal coronary angiography 1 month prior, and the last patient having had normal coronary angiography 1 year prior.

Table 1 describes the baseline characteristics of the study groups. There were no differences in age at transplant, gender, proportion of congenital heart disease versus cardiomyopathy diagnosis, or time to CAV diagnosis. Time to death or retransplant was not statistically different between the SD and HFD/retransplant groups (Figure 2).

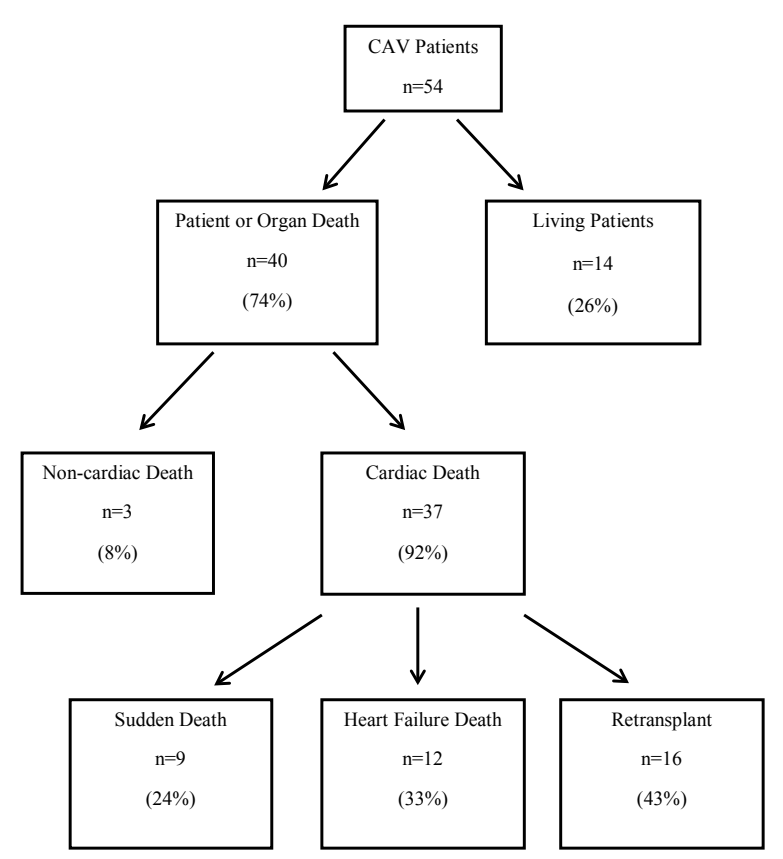

CAV: Cardiac Allograft Vasculopathy

Figure 1. Modes of death in CAV patients.

Table 1. Patient characteristics

\begin{tabular}{|l|c|c|c|}
\hline & $\begin{array}{c}\text { Sudden Death } \\
\mathbf{n = 9}\end{array}$ & $\begin{array}{c}\text { Heart Failure Death/ } \\
\text { Retransplant n=28 }\end{array}$ & $\boldsymbol{p}$-value \\
\hline Age at Transplant (years) & $\begin{array}{c}1.9 \\
(0.3-19.9)\end{array}$ & $\begin{array}{c}3.3 \\
(0.6-14.5)\end{array}$ & 0.08 \\
\hline Male & $6(67 \%)$ & $11(39 \%)$ & 0.147 \\
\hline $\begin{array}{l}\text { Congenital Heart Disease } \\
\text { Cardiomyopathy }\end{array}$ & $5(56 \%)$ & $13(46 \%)$ & 0.462 \\
\hline $\begin{array}{l}\text { Time to CAV Diagnosis } \\
\text { (years) }\end{array}$ & $4(44 \%)$ & $15(54 \%)$ & \\
\hline $\begin{array}{l}\text { Time from CAV Diagnosis to } \\
\text { Death/Retransplant (years) }\end{array}$ & $\begin{array}{c}7.0 \\
(0.2-13.1)\end{array}$ & $\begin{array}{c}5.3 \\
(1.0-16.8)\end{array}$ & 0.167 \\
\hline
\end{tabular}

$\mathrm{CAV}$, cardiac allograft vasculopathy

Continuous data are presented as median (range) and categorical data as number (\%).

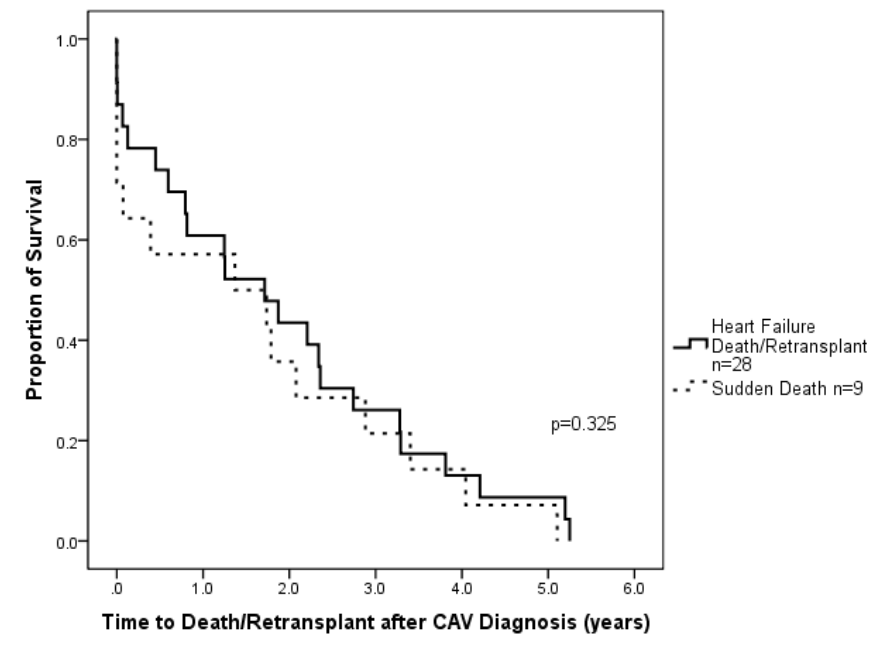

Figure 2. Survival after CAV diagnosis stratified by mode of death. (Kaplan-Meier survival analysis of CAV patients who died suddenly $(n=9)$ compared to those who died of heart failure or underwent retransplantation for heart failure $(n=28)$. The sharp decline at time 0 reflects those patients who were diagnosed with CAV by post mortem examination). 


\section{Rejection episodes}

Comparison of the number of episodes of rejection per patient between the SD group and the HFD/retransplant group found no differences in the number of episodes of acute cellular rejection (ACR) or antibody mediated rejection (AMR). The median number of ACR episodes per patient in the SD group was 0 (range 0-6 episodes per patient) and the median number of ACR episodes per patient in the HFD/retransplant group was 2 (range 0-8 episodes per patient) $(\mathrm{p}=0.256)$. No patients in the SD group had any AMR episodes while the median number of AMR in the HFD/retransplant group was 0 (range 0-7 episodes per patient) $(p=0.218)$.

\section{Functional assessment by echocardiogram}

Evaluation of each patient's most recent echocardiogram prior to death or retransplant in the SD group and HFD/retransplant group found significant differences between ejection fraction (EF) with a median $\mathrm{EF}$ of $62 \%$ (range $50-70 \%$ ) in the SD group and a median EF of $50 \%$ (range $24-75 \%)$ in the HFD/retransplant group $(p=0.045)$. Some patients in the HFD/retransplant group had heart failure with preserved EF.

\section{Hemodynamic assessment by cardiac catheterization}

Comparison of the hemodynamics at the most recent cardiac catheterization was performed between the SD group and the HFD/ retransplant group (Table 2). While there were no statistically significant differences in right ventricular end diastolic pressure (RVEDP) and pulmonary capillary wedge pressure (PCWP) at CAV diagnosis, there were significant differences between the SD group and HFD/ retransplant group at their most recent cardiac catheterization prior to death with the SD group having lower filling pressures compared to the $\mathrm{HFD} /$ retransplant group. The median RVEDP of the SD group at their last cardiac catheterization was $8 \mathrm{mmHg}$ (range 3-16 $\mathrm{mmHg}$ ) versus 14 $\mathrm{mmHg}$ (range 4-24 mmHg) in the HFD/retransplant group ( $p=0.011$ ). The median PCWP of the SD group at their last cardiac catheterization was $9 \mathrm{mmHg}$ (4-16 $\mathrm{mmHg}$ ) versus $14 \mathrm{mmHg}$ (range $7-27 \mathrm{mmHg}$ ) in the HFD/retransplant group ( $p=0.027$ ).

\section{Serum biomarkers}

BNP levels and serum creatinine prior to death or retransplant were compared in the SD and HFD/retransplant group. There was a significant difference in the median BNP with a median of $260 \mathrm{pg} / \mathrm{ml}$ (range $81-953 \mathrm{pg} / \mathrm{ml}$ ) in the SD group versus $1081 \mathrm{pg} / \mathrm{ml}$ (range 207$5258 \mathrm{pg} / \mathrm{ml}$ ) in the HFD/retransplant group $(p=0.013)$. However, no significant difference was found in serum creatinine between the two groups with a median serum creatinine of $1.51 \mathrm{mg} / \mathrm{dl}$ (range 1.21-2.10 $\mathrm{mg} / \mathrm{dl}$ ) in the SD group and a median serum creatinine of 1.30 (range $0.50-2.10 \mathrm{mg} / \mathrm{dl}$ ) in the $\mathrm{HFD} /$ retransplant group.

Table 2. Hemodynamic assessment by cardiac catheterization.

\begin{tabular}{|l|c|c|c|}
\hline & $\begin{array}{c}\text { Sudden Death } \\
\mathbf{n = 9}\end{array}$ & $\begin{array}{c}\text { Heart Failure Death/ } \\
\text { Retransplant } \mathbf{n = 2 8}\end{array}$ & $\boldsymbol{p}$-value \\
\hline $\begin{array}{l}\text { RVEDP at CAV Diagnosis } \\
\text { (mmHg) }\end{array}$ & $\begin{array}{c}8 \\
(4-22)\end{array}$ & $\begin{array}{c}10 \\
(4-24)\end{array}$ & 0.249 \\
\hline Last RVEDP (mmHg) & $\begin{array}{c}8 \\
(3-16)\end{array}$ & $\begin{array}{c}14 \\
(4-24)\end{array}$ & 0.011 \\
\hline $\begin{array}{l}\text { PCWP at CAV Diagnosis } \\
\text { (mmHg) }\end{array}$ & $\begin{array}{c}10 \\
(5-20)\end{array}$ & $\begin{array}{c}13 \\
(5-24)\end{array}$ & 0.215 \\
\hline Last PCWP(mmHg) & 9 & 14 & 0.027 \\
\hline
\end{tabular}

RVEDP: Right Ventricular End Diastolic Pressure; CAV: Cardiac Allograft Vasculopathy; PCWP: Pulmonary Capillary Wedge Pressure

\section{Arrhythmias and Implantable Cardioverter-Defibrillator (ICD)/Pacemaker use}

Comparison of the SD group to the HFD/retransplant group did not find any differences in the proportion of patients who had previous atrial or ventricular tachyarrhythmias on Holter monitoring $(p=0.454)$. Holter monitoring data were available for all the SD patients and 22/28 of the HFD/retransplant patients. Table 3 describes the proportion of patients within the two groups who had documented atrial tachycardia, ventricular tachycardia, or both on Holter monitoring.

Of the 9 patients in the SD group, 1 patient (11\%) had an ICD (indicated for primary prevention) and 1 (11\%) patient had a pacemaker (indicated for bradycardia secondary to antiarrhythmic agent used for treatment of atrial flutter and atrial tachycardia) implanted prior to death. Among the 28 patients in the HFD/retransplant group, six of them had ICDs (21\%) and four of them had pacemakers (14\%). The indications for ICD placement included primary prevention, history of nonsustained ventricular tachycardia, and syncope while the indications for pacemaker placement included second degree atrioventricular block, complete atrioventricular block, sinus node dysfunction, and syncope. When comparing usage of ICDs and pacemakers between the SD group and the HFD/retransplant group, there were no significant differences $(p=0.682)$. No patients in either group received appropriate or inappropriate shocks by their ICDs at the time of death, of those devices that were able to be interrogated.

\section{Conclusions}

This is the first pediatric study to assess characteristics of those patients who experience sudden cardiac death with a diagnosis of cardiac allograft vasculopathy. The proportion of sudden death in our study cohort, $24 \%$, is similar to prior studies, where sudden death was reported in $16-29 \%$ of heart transplant deaths $[7,8,10]$. To our knowledge, the hemodynamic differences we have shown between the SD group and the HFD/retransplant group are the first to be published in the literature. There have been other single center studies looking at cardiac catheterization data in pediatric heart transplant patients, demonstrating that patients with CAV have higher filling pressures with a mean RVEDP of $9.5 \mathrm{mmHg}$ and a mean PCWP of $12.9 \mathrm{mmHg}$ in one study and a mean RVEDP of $11 \mathrm{mmHg}$ and a mean PCWP of $14 \mathrm{mmHg}$ in the other study, but correlations with sudden death were not assessed [11,12]. The recent Pediatric Heart Transplant Study registry also assessed hemodynamics and echocardiographic assessment of systolic function as it related to graft loss, but again, that study did not delineate the modes of death associated with graft loss [8]. In comparison, our study found a lower RVEDP and PCWP as well as higher EF in the SD patients compared to the HFD/retransplant patients. The finding of lower filling pressures in the SD patients in our study demonstrates the need to consider retransplantation in those CAV patients who may not have the classic indication of heart failure. In the study by Price et al, patients with CAV and sudden death events were more likely to have symptom complexes of chest and

Table 3. Arrhythmias detected by Holter monitor.

\begin{tabular}{|l|c|c|c|}
\hline & $\begin{array}{c}\text { Sudden Death } \\
\mathbf{n = 9}\end{array}$ & $\begin{array}{c}\text { Heart Failure } \\
\text { Death/Retransplant } \\
\mathbf{n = 2 2}\end{array}$ & \multirow{2}{*}{-value } \\
\hline Atrial Tachycardia & $3(33 \%)$ & $4(18 \%)$ & \multirow{2}{*}{0.483} \\
\hline Ventricular Tachycardia & $1(11 \%)$ & $5(23 \%)$ & \\
\cline { 1 - 2 } $\begin{array}{l}\text { Both Atrial and Ventricular } \\
\text { Tachycardia }\end{array}$ & 0 & $3(14 \%)$ & \\
\hline No Arrhythmias & $5(56 \%)$ & $10(45 \%)$ & \\
\hline
\end{tabular}


abdominal pain [13]. Identifying symptom complexes may be useful to help further stratify their risk and need for retransplantation despite reassuring hemodynamics.

In addition to hemodynamic differences between the SD group and the HFD/retransplant group, we also identified a significant difference in BNP levels with the SD group having a lower median BNP of $260 \mathrm{pg} /$ $\mathrm{ml}$ versus a median of $1081 \mathrm{pg} / \mathrm{ml}$ in the HFD/retransplant group. This finding correlates with the findings of differences in filling pressures and systolic function between the two groups. The lower BNP in the SD group contrasts with an adult study showing that higher BNP levels were associated with sudden cardiac death and ventricular tachycardia in non-transplanted patients with heart failure with reduced EF [14]. Our study demonstrated that both the SD patients and HFD/ retranplant patients did have episodes of nonsustained ventricular tachycardia, but despite having differences in BNP levels, there were no differences in the proportion of patients who experienced ventricular tachycardia, which may be due to the small sample size.

While we found hemodynamic differences between the SD group and the HFD/retransplant, we did not find any differences in the number of rejection episodes per patient. This differs from prior studies in both adult and pediatric heart transplant recipients, which have shown an association between acute cellular rejection and sudden death $[7,10,15]$. Our study may not have found an association between rejection episodes and sudden death given our small sample size and the fact that our cohort as a whole did not have a high prevalence of either acute cellular or antibody mediated rejection.

Lastly, we found no differences in the use of ICDs and pacemakers between the SD group and HFD/retransplant group. The two patients in the SD group with devices died suddenly despite having a functional ICD or pacemaker. This is likely because their terminal rhythm was asystole or pulseless electrical activity with a myocardium that could not respond to pacing. This would be consistent with previously published adult studies $[5,6]$.

This study has limitations due to the small sample size and retrospective nature of data collection and analysis. Given the small sample size, we were limited in our statistical analysis and conclusions that could be drawn from them. We also chose not to categorize the degree of CAV as previous multicenter studies have done given the already small sample of patients in the groups $[16,17]$. Because our inclusion criteria required the diagnosis of CAV to be made by angiography or pathology, we may not have included patients who have microvascular disease that is difficult to detect by angiography, a known limitation of this gold standard diagnostic tool [18-20]. Despite these limitations, we were able to demonstrate that a significant number of children with CAV die suddenly. Pediatric patients who died suddenly were more likely to have better hemodynamics at catheterization and normal systolic function by echocardiogram, as well as lower BNP levels. Multicenter investigation of the CAV population is warranted to determine if a different set of criteria should be used to relist those patients who are at risk of sudden death and do not necessarily have obvious graft failure. Ultimately, clinicians should not be overly reassured by normal hemodynamics and relatively low BNP levels in the setting of CAV.

\section{Acknowledgements}

This work was supported by the Cardiovascular Clinical Research Core at Baylor College of Medicine, Texas Children's Hospital, Houston, Texas.

\section{Authors' contributions}

B.J. Hong and S.W. Denfield: Concept and study design, data analysis/interpretation, drafting manuscript; S. K. Sexson Tejtel: study design and data analysis; A. Jeewa, A.G. Cabrera, J.F. Price, J.S. Heinle, W.J. Dreyer: manuscript revision and approval.

\section{Disclosures}

None of the authors have conflicts of interest to disclose.

\section{References}

1. Dipchand AI, Kirk R, Edwards LB (2013) The Registry of the International Society for Heart and Lung Transplantation: sixteenth official pediatric heart transplantation report - 2013. J Heart Lung Transplant 32: 979-88.

2. Canter CE, Shaddy RE, Bernstein D (2007) Indications for Heart Transplantation in Pediatric Heart Disease. Circulation 115: 658-76.

3. Chantranuwat C, Blakey JD, Kobashigawa JA, Moriguchi JD, Laks H, et al. (2004) Sudden, unexpected death in cardiac transplant recipients: an autopsy study. $J$ Heart Lung Transplant 23: 683-689. [Crossref]

4. Shivkumar K, Espejo M, Kobashigawa J, Watanabe M, Ikeda M, et al. (2001) Sudden death after heart transplantation: the major mode of death. J Heart Lung Transplant 20: 180. [Crossref]

5. Vaseghi M, Lellouche N, Ritter H, Fonarow GC, Patel JK, et al. (2009) Mode and mechanisms of death after orthotopic heart transplantation. Heart Rhythm 6: 503-509. [Crossref]

6. Tsai VW, Cooper J, Garan H (2009) The efficacy of implantable cardioverterdefibrillator in heart transplant recipients: results from a multicenter registry. Circulation Heart Failure 2: 197-201.

7. Daly KP, Chakravarti SB, Tresler M (2011) Sudden death after pediatric heart transplantation: Analysis of data from the Pediatric Heart Transplant Study Group. $J$ Heart Lung Transplant 30: 1395-1402.

8. Kindel SJ, Law YM, Chin C (2015) Improved Detection of Cardiac Allograft Vasculopathy: A multi-institutional analysis of functional parameters in pediatric heart transplant recipients. J Heart Lung Transplant 55: 547-57.

9. McAllister HA Jr, Schnee MJ, RadovanceviÄ $\ddagger$ B, Frazier OH (1986) A system for grading cardiac allograft rejection. Tex Heart Inst J 13: 1-3. [Crossref]

10. Zuppan CW, Wells LM, Kerstetter JC, (2009) Cause of death in pediatric and infant heart transplant recipients: review of a 20 -year, single-institution cohort. J Heart Lung Transplant 28: 579-84.

11. Aiyagari R, Nika M, Gurney JG (2011) Association of pediatric heart transplant coronary vasculopathy with abnormal hemodynamic measures. Congenital Heart Disease 6: 128-33

12. Law Y, Boyle G, Miller S, Clendaniel J, Ettedgui J, et al. (2006) Restrictive hemodynamics are present at the time of diagnosis of allograft coronary artery disease in children. Pediatr Transplant 10: 948-952. [Crossref]

13. Price JF, Towbin JA, Dreyer WJ (2005) Symptom complex is associated with transplant coronary artery disease and sudden death/resuscitated sudden death in pediatric heart transplant recipients. J Heart Lung Transplant 24: 1798-803.

14. Levine YC, Rosenberg MA, Mittleman M, Samuel M, Methachittiphan N, et al. (2014) B-type natriuretic peptide is a major predictor of ventricular tachyarrhythmias. Heart Rhythm 11: 1109-1116. [Crossref]

15. Herre JM, Brown RN, Chang PP (2007) Sudden cardiac death after heart transplant: Insights from the cardiac transplant research database. J Heart Lung Transplant 26: 339 .

16. Pahl E, Naftel DC, Kuhn MA, Shaddy RE, Morrow WR, et al. (2005) The impact and outcome of transplant coronary artery disease in a pediatric population: a 9-year multiinstitutional study. J Heart Lung Transplant 24: 645-651. [Crossref]

17. Costello JM, Wax DF, Bins JH (2003) A comparison of intravascular ultrasound with coronary angiography for evaluation of transplant coronary artery disease in pediatric heart transplant recipients. J Heart Lung Transplant 22: 44-9.

18. Kuhn MA, Jutzy KR, Deming DD (2000) The medium-term findings in coronary arteries by intravascular ultrasound in infants and children after heart transplantation. JACC 36: 250-4. 
19. Johnson DE, Alderman EL, Schroeder JS (1991) Transplant coronary artery disease: histopathologic correlations with angiographic morphology. JACC 17: 449-57.
20. Mulla NF, Johnston JK, Vander Dussen L, Beeson WL, Chinnock RE, et al. (2001) Late rejection is a predictor of transplant coronary artery disease in children. $J$ Am Coll Cardiol 37: 243-250. [Crossref]

Copyright: $(02016$ Hong BJ. This is an open-access article distributed under the terms of the Creative Commons Attribution License, which permits unrestricted use, distribution, and reproduction in any medium, provided the original author and source are credited. 